\title{
Convergence of positive series and ideal convergence* $^{*}$
}

\section{Vladimír Baláž ${ }^{a}$, Kálmán Liptai ${ }^{b}$, János T. Tóth $^{c}$, Tomáš Visnyai ${ }^{a}$}

${ }^{a}$ Institute of Information Engineering, Automation and Mathematics, Faculty of Chemical and Food Technology, University of Technology in Bratislava, Radlinského 9, 81237 Bratislava, Slovakia vladimir.balaz@stuba.sk tomas.visnyai@stuba.sk

${ }^{b}$ Department of Applied Mathematics, Eszterházy Károly University, Leányka 4 3300 Eger, Hungary liptai.kalman@uni-eszterhazy.hu

${ }^{c}$ Department of Mathematics, J. Selye University, P. O. Box 54, 94501 Komárno, Slovakia tothj@ujs.sk

Submitted: May 13, 2020

Accepted: May 30, 2020

Published online: June 15, 2020

\begin{abstract}
Let $\mathcal{I} \subseteq 2^{\mathbb{N}}$ be an admissible ideal, we say that a sequence $\left(x_{n}\right)$ of real numbers $\mathcal{I}$-converges to a number $L$, and write $\mathcal{I}-\lim x_{n}=L$, if for each $\varepsilon>0$ the set $A_{\varepsilon}=\left\{n:\left|x_{n}-L\right| \geq \varepsilon\right\}$ belongs to the ideal $\mathcal{I}$. In this paper we discuss the relation ship between convergence of positive series and the convergence properties of the summand sequence. Concretely, we study the ideals $\mathcal{I}$ having the following property as well:

$$
\sum_{n=1}^{\infty} a_{n}^{\alpha}<\infty \text { and } 0<\inf _{n} \frac{n}{b_{n}} \leq \sup _{n} \frac{n}{b_{n}}<\infty \Rightarrow \mathcal{I}-\lim a_{n} b_{n}^{\beta}=0,
$$

\footnotetext{
${ }^{*}$ This contribution was partially supported by The Slovak Research and Development Agency under the grant VEGA No. 2/0109/18.
} 
where $0<\alpha \leq 1 \leq \beta \leq \frac{1}{\alpha}$ are real numbers and $\left(a_{n}\right),\left(b_{n}\right)$ are sequences of positive real numbers. We characterize $T\left(\alpha, \beta, a_{n}, b_{n}\right)$ the class of all such admissible ideals $\mathcal{I}$.

This accomplishment generalized and extended results from the papers $[4,7,12,16]$, where it is referred that the monotonicity condition of the summand sequence in so-called Olivier's Theorem (see [13]) can be dropped if the convergence of the sequence $\left(n a_{n}\right)$ is weakend. In this paper we will study $\mathcal{I}$-convergence mainly in the case when $\mathcal{I}$ stands for $\mathcal{I}_{<q}, \mathcal{I}_{c}^{(q)}, \mathcal{I}_{\leq q}$, respectively.

Keywords: $\mathcal{I}$-convergence, convergence of positive series, Olivier's theorem, admissible ideals, convergence exponent

$M S C: 40 \mathrm{~A} 05,40 \mathrm{~A} 35$

\section{Introduction}

We recall the basic definitions and conventions that will be used throughout the paper. Let $\mathbb{N}$ be the set of all positive integers. A system $\mathcal{I}, \emptyset \neq \mathcal{I} \subseteq 2^{\mathbb{N}}$ is called an ideal, provided $\mathcal{I}$ is additive $(A, B \in \mathcal{I}$ implies $A \cup B \in \mathcal{I})$, and hereditary $(A \in \mathcal{I}, B \subset A$ implies $B \in \mathcal{I})$. The ideal is called nontrivial if $\mathcal{I} \neq 2^{\mathbb{N}}$. If $\mathcal{I}$ is a nontrivial ideal, then $\mathcal{I}$ is called admissible if it contains the singletons $(\{n\} \in \mathcal{I}$ for every $n \in \mathbb{N}$ ). The fundamental notation which we shall use is $\mathcal{I}$-convergence introduced in the paper [11] ( see also [3] where $\mathcal{I}$-convergence is defined by means of filter-the dual notion to ideal). The notion $\mathcal{I}$-convergence corresponds to the natural generalization of the notion of statistical convergence ( see $[5,17]$ ).

Definition 1.1. Let $\left(x_{n}\right)$ be a sequence of real (complex) numbers. We say that the sequence $\mathcal{I}$-converges to a number $L$, and write $\mathcal{I}-\lim x_{n}=L$, if for each $\varepsilon>0$ the set $A_{\varepsilon}=\left\{n:\left|x_{n}-L\right| \geq \varepsilon\right\}$ belongs to the ideal $\mathcal{I}$.

In the following we suppose that $\mathcal{I}$ is an admissible ideal. Then for every sequence $\left(x_{n}\right)$ we have immediately that $\lim _{n \rightarrow \infty} x_{n}=L$ (classic limit) implies that $\left(x_{n}\right)$ also $\mathcal{I}$-converges to a number $L$. Let $\mathcal{I}_{f}$ be the ideal of all finite subsets of $\mathbb{N}$. Then $\mathcal{I}_{f}$-convergence coincides with the usual convergence. Let $\mathcal{I}_{d}=\{A \subseteq \mathbb{N}: d(A)=0\}$, where $d(A)$ is the asymptotic density of $A \subseteq \mathbb{N}$ $\left(d(A)=\lim _{n \rightarrow \infty} \frac{\#\{a \leq n: a \in A\}}{n}\right.$, where $\# M$ denotes the cardinality of the set $\left.M\right)$. Usual $\mathcal{I}_{d}$-convergence is called statistical convergence. For $0<q \leq 1$ the class

$$
\mathcal{I}_{c}^{(q)}=\left\{A \subset \mathbb{N}: \sum_{a \in A} a^{-q}<\infty\right\}
$$

is an admissible ideal and whenever $0<q<q^{\prime}<1$, we get

$$
\mathcal{I}_{f} \subsetneq \mathcal{I}_{c}^{(q)} \subsetneq \mathcal{I}_{c}^{q^{\prime}} \subsetneq \mathcal{I}_{c}^{(1)} \subsetneq \mathcal{I}_{d}
$$

The notions the admissible ideal and $\mathcal{I}$-convergence have been developed in several directions and have been used in various parts of mathematics, in particular in 
number theory, mathematical analysis and ergodic theory, for example $[1,2,5,6$, $9-11,15,17-19]$.

Let $\lambda$ be the convergence exponent function on the power set of $\mathbb{N}$, thus for $A \subset \mathbb{N}$ put

$$
\lambda(A)=\inf \left\{t>0: \sum_{a \in A} a^{-t}<\infty\right\} .
$$

If $q>\lambda(A)$ then $\sum_{a \in A} \frac{1}{a^{q}}<\infty$, and $\sum_{a \in A} \frac{1}{a^{q}}=\infty$ when $q<\lambda(A)$; if $q=\lambda(A)$, the convergence of $\sum_{a \in A} \frac{1}{a^{q}}$ is inconclusive. It follows from [14, p. 26, Examp. 113, 114] that the range of $\lambda$ is the interval [0,1], moreover for $A=\left\{a_{1}<a_{2}<\cdots<\right.$ $\left.a_{n}<\ldots\right\} \subseteq \mathbb{N}$ the convergence exponent can be calculate by using the following formula

$$
\lambda(A)=\limsup _{n \rightarrow \infty} \frac{\log n}{\log a_{n}} .
$$

It is easy to see that $\lambda$ is monotonic, i.e. $\lambda(A) \leq \lambda(B)$ whenever $A \subseteq B \subset \mathbb{N}$, furthermore, $\lambda(A \cup B)=\max \{\lambda(A), \lambda(B)\}$ for all $A, B \subset \mathbb{N}$.

\section{Overwiew of known results}

In this section we mention known results related to the topic of this paper and some other ones we use in the proofs of our results. Recently in [19] was introduced the following classes of subsets of $\mathbb{N}$ :

$$
\begin{aligned}
\mathcal{I}_{<q} & =\{A \subset \mathbb{N}: \lambda(A)<q\}, \text { if } 0<q \leq 1, \\
\mathcal{I}_{\leq q} & =\{A \subset \mathbb{N}: \lambda(A) \leq q\}, \text { if } 0 \leq q \leq 1, \text { and } \\
\mathcal{I}_{0} & =\{A \subset \mathbb{N}: \lambda(A)=0\} .
\end{aligned}
$$

Clearly, $\mathcal{I}_{\leq 0}=\mathcal{I}_{0}$. Since $\lambda(A)=0$ when $A \subset \mathbb{N}$ is finite, then $\mathcal{I}_{f}=\{A \subset \mathbb{N}$ : $A$ is finite $\} \subset \mathcal{I}_{0}$, moreover, there is proved [19, Th.2] that each class $\mathcal{I}_{0}, \mathcal{I}_{<q}, \mathcal{I}_{\leq q}$, respectively forms an admissible ideal, except for $\mathcal{I}_{\leq 1}=2^{\mathbb{N}}$.

Proposition 2.1 ([19, Th.1]). Let $0<q<q^{\prime}<1$. Then we have

$$
\mathcal{I}_{0} \subsetneq \mathcal{I}_{<q} \subsetneq \mathcal{I}_{c}^{(q)} \subsetneq \mathcal{I}_{\leq q} \subsetneq \mathcal{I}_{<q^{\prime}} \subsetneq \mathcal{I}_{c}^{\left(q^{\prime}\right)} \subsetneq \mathcal{I}_{\leq q^{\prime}} \subsetneq \mathcal{I}_{<1} \subsetneq \mathcal{I}_{c}^{(1)} \subsetneq \mathcal{I}_{\leq 1}=2^{\mathbb{N}}
$$

and the difference of successive sets is infinite, so equality does not hold in any of the inclusions.

The claim in the following proposition is a trivial fact about preservation of the limit.

Proposition 2.2 ([11, Lemma]). If $\mathcal{I}_{1} \subset \mathcal{I}_{2}$, then $\mathcal{I}_{1}-\lim x_{n}=L$ implies $\mathcal{I}_{2}-$ $\lim x_{n}=L$.

In [13] L. Olivier proved results so-called Olivier's Theorem about the speed of convergence to zero of the terms of convergent positive series with nonincreasing 
terms. Precisely, if $\left(a_{n}\right)$ is a nonincreasing positive sequence and $\sum_{n=1}^{\infty} a_{n}<\infty$, then $\lim _{n \rightarrow \infty} n a_{n}=0$ (see also [8]). In [16], T. Šalát and V. Toma made the remark that the monotonicity condition in Olivier's Theorem can be dropped if the convergence the sequence $\left(n a_{n}\right)$ is weakened by means of the notion of $\mathcal{I}$ convergence (see also [7]). In [12], there is an extension of results in [16] with very nice historical contexts of the object of our research.

Since $0=\lim _{n \rightarrow \infty} n a_{n}=\mathcal{I}_{f}-\lim n a_{n}$, then the above mentioned Olivier's Theorem can be formulated in the terms of $\mathcal{I}$-convergence as follows:

$$
\left(a_{n}\right) \text { nonincreasing and } \sum_{n=1}^{\infty} a_{n}<\infty \Rightarrow \mathcal{I}-\lim n a_{n}=0 \text {, }
$$

holds for any admissible ideal $\mathcal{I}$ (this assertion is a direct corollary of the facts $\mathcal{I}_{f} \subseteq \mathcal{I}$ and Proposition 2.2), and providing $\left(a_{n}\right)$ to be a sequence of positive real numbers.

The following simple example

$$
a_{n}= \begin{cases}\frac{1}{n}, & \text { if } n=k^{2},(k=1,2, \ldots) \\ \frac{1}{2^{n}}, & \text { otherwise }\end{cases}
$$

shows that monotonicity condition of the positive sequence $\left(a_{n}\right)$ can not be in general omitted. This example shows that $\limsup _{n \rightarrow \infty} n a_{n}=1$, thus the ideal $\mathcal{I}_{f}$ does not have for positive terms the following property

$$
\sum_{n=1}^{\infty} a_{n}<\infty \Rightarrow \mathcal{I}-\lim n a_{n}=0 .
$$

The previous example can be strengthened taking $a_{n}=\frac{\log n}{n}$ if $n$ is square, in such case the sequence $\left(n a_{n}\right)$ is not bounded yet. In [16], T. Šalát and V. Toma characterized the class $S(T)$ of all admissible ideals $\mathcal{I} \subset 2^{\mathbb{N}}$ having the property $(2.1)$, for sequences $\left(a_{n}\right)$ of positive real numbers.

They proved that

$$
S(T)=\left\{\mathcal{I} \subset 2^{\mathbb{N}}: \mathcal{I} \text { is an admissible ideal such that } \mathcal{I} \supseteq \mathcal{I}_{c}^{(1)}\right\} .
$$

J. Gogola, M. Mačaj, T. Visnyai in [7] introduced and characterized the class $S_{q}(T)$ of all admissible ideals $\mathcal{I} \subset 2^{\mathbb{N}}$ for $0<q \leq 1$ having the property

$$
\sum_{n=1}^{\infty} a_{n}^{q}<\infty \Rightarrow \mathcal{I}-\lim n a_{n}=0
$$

providing $\left(a_{n}\right)$ be a positive real sequence. The stronger condition of convergence of positive series requirest the stronger convergence property of the summands as well. They proved

$$
S_{q}(T)=\left\{\mathcal{I} \subset 2^{\mathbb{N}}: \mathcal{I} \text { is an admissible ideal such that } \mathcal{I} \supseteq \mathcal{I}_{c}^{(q)}\right\} .
$$


Of course, if $q=1$ then $S_{1}(T)=S(T)$.

In [12], C. P. Niculescu, G. T. Prăjitură studied the following implication, which is general as $(2.1)$ :

$$
\sum_{n=1}^{\infty} a_{n}<\infty \text { and } \inf _{n} \frac{n}{b_{n}}>0 \Rightarrow \mathcal{I}-\lim a_{n} b_{n}=0
$$

for sequences $\left(a_{n}\right),\left(b_{n}\right)$ of positive real numbers.

They proved that the ideal $\mathcal{I}_{d}$ fulfills $(2.3)$. In the next section we are going to show that $\mathcal{I}_{c}^{(1)}$ is the smallest admissible ideal partially ordered by inclusion which also fulfills $(2.3)$.

\section{3. $\mathcal{I}_{c}^{(q)}-$ convergence and convergence of positive se- ries}

In this part we introduce and characterize the class of such ideals that fulfill the following implication (3.1). Obviously this class will generalize the results of $(2.2)$ and (2.3). On the other hand, we define the smallest admissible ideal partially ordered by inclusion which fulfills (3.1).

In the sequel we are going to study the ideals $\mathcal{I}$ having the following property:

$$
\sum_{n=1}^{\infty} a_{n}^{\alpha}<\infty \text { and } 0<\inf _{n} \frac{n}{b_{n}} \leq \sup _{n} \frac{n}{b_{n}}<\infty \Rightarrow \mathcal{I}-\lim a_{n} b_{n}^{\beta}=0
$$

where $0<\alpha \leq 1 \leq \beta \leq \frac{1}{\alpha}$ are real numbers and $\left(a_{n}\right),\left(b_{n}\right)$ are positive sequences of real numbers.

We denote by $T\left(\alpha, \beta, a_{n}, b_{n}\right)$ the class of all admissible ideals $\mathcal{I} \subset 2^{\mathbb{N}}$ having the property (3.1). Obviously $T\left(1,1, a_{n}, n\right)=S(T)$ and $T\left(q, 1, a_{n}, n\right)=S_{q}(T)$.

Theorem 3.1. Let $0<\alpha \leq 1 \leq \beta \leq \frac{1}{\alpha}$ be real numbers. Then for every positive real sequences $\left(a_{n}\right),\left(b_{n}\right)$ such that

$$
\sum_{n=1}^{\infty} a_{n}^{\alpha}<\infty \quad \text { and } \quad \inf _{n} \frac{n}{b_{n}}>0
$$

we have

$$
\mathcal{I}_{c}^{(\alpha \beta)}-\lim a_{n} b_{n}^{\beta}=0 .
$$

Proof. Let $\varepsilon>0$, put $A_{\varepsilon}=\left\{n \in \mathbb{N}: a_{n} b_{n}^{\beta} \geq \varepsilon\right\}$. We proceed by contradiction. Then there exists such $\varepsilon>0$ that $A_{\varepsilon} \notin \mathcal{I}_{c}^{(\alpha \beta)}$, thus

$$
\sum_{n \in A_{\varepsilon}} \frac{1}{n^{\alpha \beta}}=\infty
$$


For $n \in A_{\varepsilon}$ we have

$$
a_{n}^{\alpha} \geq \varepsilon^{\alpha} \frac{1}{b_{n}^{\alpha \beta}}=\varepsilon^{\alpha}\left(\frac{n}{b_{n}}\right)^{\alpha \beta} \frac{1}{n^{\alpha \beta}} \geq \varepsilon^{\alpha}\left(\inf _{n} \frac{n}{b_{n}}\right)^{\alpha \beta} \frac{1}{n^{\alpha \beta}},
$$

and so

$$
\sum_{n=1}^{\infty} a_{n}^{\alpha} \geq \sum_{n \in A_{\varepsilon}} a_{n}^{\alpha} \geq \varepsilon^{\alpha}\left(\inf _{n} \frac{n}{b_{n}}\right)^{\alpha \beta} \sum_{n \in A_{\varepsilon}} \frac{1}{n^{\alpha \beta}} .
$$

Using this and the assumption for a sequence $\left(b_{n}\right)$ and $(3.2)$ we get

$$
\sum_{n=1}^{\infty} a_{n}^{\alpha}=\infty
$$

which is a contradiction.

If in Theorem 3.1 we put $\alpha=q$ and $\beta=1$, we can obtain the following corollary.

Corollary 3.2. For every positive real sequences $\left(a_{n}\right),\left(b_{n}\right)$ such that

$$
\sum_{n=1}^{\infty} a_{n}^{q}<\infty \quad \text { and } \quad \inf _{n} \frac{n}{b_{n}}>0
$$

we have

$$
\mathcal{I}_{c}^{(q)}-\lim a_{n} b_{n}=0 .
$$

Already in the case when $q=1$ in Corollary 3.2, we get a stronger assertion than given in [12] for the ideal $\mathcal{I}_{d}$, because of $\mathcal{I}_{c}^{(1)} \subsetneq \mathcal{I}_{d}$.

Remark 3.3. Let $\left(a_{n}\right),\left(b_{n}\right)$ be positive real sequences. For special choices $\alpha$ and $\left(b_{n}\right)$ in Corollary 3.2 , we can obtain the following:

i) Putting $\alpha=1$. Then we get: If $\sum_{n=1}^{\infty} a_{n}<\infty$ and $\inf _{n} \frac{n}{b_{n}}>0$ then $\mathcal{I}_{c}^{(1)}-$ $\lim a_{n} b_{n}=0$ ( which is stronger result as $[12$, Theorem 5$]$ ).

ii) Putting $\alpha=1$ and $b_{n}=n$. Then we get: If $\sum_{n=1}^{\infty} a_{n}<\infty$ then $\mathcal{I}_{c}^{(1)}-$ $\lim a_{n} n=0($ see $[16$, Theorem 2.1]).

iii) Putting $\alpha=q$ and $b_{n}=n$. Then we get: If $\sum_{n=1}^{\infty} a_{n}^{q}<\infty$ then $\mathcal{I}_{c}^{(q)}-$ $\lim a_{n} n=0($ see $[7$, Lemma 3.1]).

Theorem 3.4. Let $0<\alpha \leq 1 \leq \beta \leq \frac{1}{\alpha}$ be real numbers. If for some admissible ideal $\mathcal{I}$ holds

$$
\mathcal{I}-\lim a_{n} b_{n}^{\beta}=0
$$

for every sequences $\left(a_{n}\right),\left(b_{n}\right)$ of positive numbers such that

$$
\sum_{n=1}^{\infty} a_{n}^{\alpha}<\infty \quad \text { and } \sup _{n} \frac{n}{b_{n}}<\infty
$$

then

$$
\mathcal{I}_{c}^{(\alpha \beta)} \subseteq \mathcal{I}
$$


Proof. Let us assume that for some admissible ideal $\mathcal{I}$ we have $\mathcal{I}-\lim a_{n} b_{n}^{\beta}=0$ and take an arbitrary set $M \in \mathcal{I}_{c}^{(\alpha \beta)}$. It is sufficient to prove that $M \in \mathcal{I}$. Since $\mathcal{I}-\lim a_{n} b_{n}^{\beta}=0$ we have for each $\varepsilon>0$ the set $A_{\varepsilon}=\left\{n \in \mathbb{N}: a_{n} b_{n}^{\beta} \geq \varepsilon\right\} \in \mathcal{I}$. Since $M \in \mathcal{I}_{c}^{(\alpha \beta)}$ we have $\sum_{n \in M} \frac{1}{n^{\alpha \beta}}<\infty$. Now we define the sequence $a_{n}$ as follows:

$$
a_{n}= \begin{cases}\frac{1}{n^{\beta}}, & \text { if } n \in M \\ \frac{1}{2^{n}}, & \text { if } n \notin M\end{cases}
$$

Obviously the sequence $\left(a_{n}\right)$ fulfills the premises of the theorem as $a_{n}>0$ and

$$
\sum_{n=1}^{\infty} a_{n}^{\alpha}=\sum_{n \in M}\left(\frac{1}{n^{\beta}}\right)^{\alpha}+\sum_{n \notin M}\left(\frac{1}{2^{n}}\right)^{\alpha} \leq \sum_{n \in M} \frac{1}{n^{\alpha \beta}}+\sum_{n=1}^{\infty}\left(\frac{1}{2^{\alpha}}\right)^{n}<\infty .
$$

Hence $a_{n} n^{\beta}=1$ for $n \in M$ and so for each $n \in M$ we have

$$
a_{n} b_{n}^{\beta}=a_{n} n^{\beta}\left(\frac{b_{n}}{n}\right)^{\beta}=\left(\frac{b_{n}}{n}\right)^{\beta} \geq \frac{1}{\left(\sup _{n} \frac{n}{b_{n}}\right)^{\beta}}>0 .
$$

Denote by $\varepsilon(\beta)=\left(\sup _{n} \frac{n}{b_{n}}\right)^{-\beta}>0$ and preceding considerations give us

$$
M \subset A_{\varepsilon(\beta)} \in \mathcal{I} \text {. }
$$

Thus $M \in \mathcal{I}$, what means $\mathcal{I}_{c}^{(\alpha \beta)} \subseteq \mathcal{I}$.

The characterization of the class $T\left(\alpha, \beta, a_{n}, b_{n}\right)$ is the direct consequence of Theorem 3.1 and Theorem 3.4.

Theorem 3.5. Let $0<\alpha \leq 1 \leq \beta \leq \frac{1}{\alpha}$ be real numbers and $\left(a_{n}\right),\left(b_{n}\right)$ be sequences of positive real numbers. Then the class $T\left(\alpha, \beta, a_{n}, b_{n}\right)$ consists of all admissible ideals $\mathcal{I} \subset 2^{\mathbb{N}}$ such that $\mathcal{I} \supseteq \mathcal{I}_{c}^{(\alpha \beta)}$.

For special choices $\alpha, \beta$ and $\left(b_{n}\right)$ in Theorem 3.5 we can get the following.

Corollary 3.6. Let $0<q \leq 1$ be a real number and $\left(a_{n}\right)$ be positive real sequences having the properties

$$
\sum_{n=1}^{\infty} a_{n}^{q}<\infty
$$

Then we have
i) $T\left(q, 1, a_{n}, n\right)=\left\{\mathcal{I} \subset 2^{\mathbb{N}}: \mathcal{I}\right.$ is admissible ideal such that $\left.\mathcal{I} \supseteq \mathcal{I}_{c}^{(q)}\right\}=S_{q}(T)$,
ii) $T\left(1,1, a_{n}, n\right)=\left\{\mathcal{I} \subset 2^{\mathbb{N}}: \mathcal{I}\right.$ is admissible ideal such that $\left.\mathcal{I} \supseteq \mathcal{I}_{c}^{(1)}\right\}=S(T)$. 


\section{4. $\mathcal{I}_{<q}-$ and $\mathcal{I}_{\leq q}-$ convergence and convergence of series}

In this section we will study the admissible ideals $\mathcal{I} \subset 2^{\mathbb{N}}$ having the special property (4.1) and (4.3), respectively.

$$
\sum_{n=1}^{\infty} a_{n}^{q_{k}}<\infty \text { for every } k \text { and } 0<\inf _{n} \frac{n}{b_{n}} \leq \sup _{n} \frac{n}{b_{n}}<\infty \Rightarrow \mathcal{I}-\lim a_{n} b_{n}=0,
$$

where $\left(q_{k}\right)$ is a strictly decreasing sequence which is convergent to $q, 0 \leq q<1$ and $\left(a_{n}\right),\left(b_{n}\right)$ are sequences of positive real numbers.

Denote by $T_{q}^{q_{k}}\left(a_{n}, b_{n}\right)$ the class of all admissible ideals $\mathcal{I}$ having the property (4.1).

Theorem 4.1. Let $0 \leq q<1$ and $\left(q_{k}\right)$ be a strictly decreasing sequence which is convergent to $q$. Then for positive real sequences $\left(a_{n}\right),\left(b_{n}\right)$ such that holds

$$
\sum_{n=1}^{\infty} a_{n}^{q_{k}}<\infty, \text { for every } k \text { and } \inf _{n} \frac{n}{b_{n}}>0
$$

we have

$$
\mathcal{I}_{\leq q}-\lim a_{n} b_{n}=0 .
$$

Proof. Again, we proceed by contradiction. Put $A_{\varepsilon}=\left\{n \in \mathbb{N}: a_{n} b_{n} \geq \varepsilon\right\}$. Then there exists such $\varepsilon>0$ that $A_{\varepsilon} \notin \mathcal{I}_{\leq q}$, thus $\lambda\left(A_{\varepsilon}\right)>q$. Hence there exists such $i \in \mathbb{N}$, that $q<q_{k_{i}}<\lambda\left(A_{\varepsilon}\right)$, and so we get

$$
\sum_{n \in A_{\varepsilon}} \frac{1}{n^{q_{k_{i}}}}=\infty
$$

For $n \in A_{\varepsilon}$ we have

$$
a_{n}^{q_{k_{i}}} \geq \varepsilon^{q_{k_{i}}} \frac{1}{b_{n}^{q_{k_{i}}}}=\varepsilon^{q_{k_{i}}}\left(\frac{n}{b_{n}}\right)^{q_{k_{i}}} \frac{1}{n^{q_{k_{i}}}} \geq \varepsilon^{q_{k_{i}}}\left(\inf _{n} \frac{n}{b_{n}}\right)^{q_{k_{i}}} \frac{1}{n^{q_{k_{i}}}},
$$

therefore

$$
\sum_{n=1}^{\infty} a_{n}^{q_{k_{i}}} \geq \sum_{n \in A_{\varepsilon}} a_{n}^{q_{k_{i}}} \geq \varepsilon^{q_{k_{i}}}\left(\inf _{n} \frac{n}{b_{n}}\right)^{q_{k_{i}}} \sum_{n \in A_{\varepsilon}} \frac{1}{n^{q_{k_{i}}}} .
$$

Using this and the assumption for a sequence $\left(b_{n}\right)$ and $(4.2)$ we get

$$
\sum_{n=1}^{\infty} a_{n}^{q_{k_{i}}}=\infty
$$

what is a contradiction. 
Theorem 4.2. Let $0 \leq q<1$ and $\left(q_{k}\right)$ be a strictly decreasing sequence which is convergent to $q$. If for some admissible ideal $\mathcal{I}$ holds

$$
\mathcal{I}-\lim a_{n} b_{n}=0
$$

for every sequences $\left(a_{n}\right),\left(b_{n}\right)$ of positive numbers such that

$$
\sum_{n=1}^{\infty} a_{n}^{q_{k}}<\infty, \text { for every } k \text { and } \sup _{n} \frac{n}{b_{n}}<\infty
$$

then

$$
\mathcal{I}_{\leq q} \subseteq \mathcal{I}
$$

Proof. Let us assume that for any admissible ideal $\mathcal{I}$ we have $\mathcal{I}-\lim a_{n} b_{n}=0$ and take an arbitrary set $M \in \mathcal{I}_{\leq q}$. It is sufficient to prove that $M \in \mathcal{I}$. Since $M \in \mathcal{I}_{\leq q}$ we have $\lambda(M) \leq q$ and so for each $q_{k}>q$ we get

$$
\sum_{n \in M} \frac{1}{n^{q_{k}}}<\infty
$$

Moreover $\mathcal{I}-\lim a_{n} b_{n}=0$ and so for each $\varepsilon>0$ the set $A_{\varepsilon}=\left\{n \in \mathbb{N}: a_{n} b_{n} \geq\right.$ $\varepsilon\} \in \mathcal{I}$. Define the sequence $\left(a_{n}\right)$ as follows:

$$
a_{n}= \begin{cases}\frac{1}{n}, & \text { if } n \in M \\ \frac{1}{2^{n}}, & \text { if } n \notin M\end{cases}
$$

The sequence $\left(a_{n}\right)$ fulfills the premises of the theorem, $a_{n}>0$ and for each $q_{k}$ we obtain

$$
\sum_{n=1}^{\infty} a_{n}^{q_{k}}=\sum_{n \in M} \frac{1}{n^{q_{k}}}+\sum_{n \notin M}\left(\frac{1}{2^{n}}\right)^{q_{k}} \leq \sum_{n \in M} \frac{1}{n^{q_{k}}}+\sum_{n=1}^{\infty}\left(\frac{1}{2^{q_{k}}}\right)^{n}<\infty .
$$

Now $a_{n} n=1$ for $n \in M$. Therefore for each $n \in M$ we have

$$
a_{n} b_{n}=a_{n} n\left(\frac{b_{n}}{n}\right)=\frac{b_{n}}{n} \geq \frac{1}{\sup _{n} \frac{n}{b_{n}}}>0 .
$$

Denote by $\varepsilon=\left(\sup _{n} \frac{n}{b_{n}}\right)^{-1}>0$ we have

$$
M \subset A_{\varepsilon} \in \mathcal{I} \text {. }
$$

Thus $M \in \mathcal{I}$, what means $\mathcal{I}_{\leq q} \subseteq \mathcal{I}$.

The above mentioned results (Theorem 4.1 and Theorem 4.2) allow us to give a characterization for the class $T_{q}^{q_{k}}\left(a_{n}, b_{n}\right)$.

Theorem 4.3. Let $0 \leq q<1$ and $\left(q_{k}\right)$ be a strictly decreasing sequence which converges to $q$. Let $\left(a_{n}\right),\left(b_{n}\right)$ be positive real sequences. Then the class $T_{q}^{q_{k}}\left(a_{n}, b_{n}\right)$ consists of all admissible ideals $\mathcal{I} \subset 2^{\mathbb{N}}$ such that $\mathcal{I} \supseteq \mathcal{I}_{\leq q}$. 
Let us consider the following property and pronounce for it analogical results as above.

$$
\sum_{n=1}^{\infty} a_{n}^{q_{k}}<\infty \text { for some } k \text { and } 0<\inf _{n} \frac{n}{b_{n}} \leq \sup _{n} \frac{n}{b_{n}}<\infty \Rightarrow \mathcal{I}-\lim a_{n} b_{n}=0,
$$

where $\left(q_{k}\right)$ is a strictly increasing sequence of positive numbers which is convergent to $q, 0<q \leq 1$ and $\left(a_{n}\right),\left(b_{n}\right)$ are sequences of positive real numbers.

Denote by $T_{q_{k}}^{q}\left(a_{n}, b_{n}\right)$ the class of all admissible ideals $\mathcal{I}$ having the property $(4.3)$.

Theorem 4.4. Let $0<q \leq 1$ and $\left(q_{k}\right)$ be a strictly increasing sequence of positive numbers which is convergent to $q$. Then for positive real sequences $\left(a_{n}\right),\left(b_{n}\right)$ such that holds

$$
\sum_{n=1}^{\infty} a_{n}^{q_{k_{0}}}<\infty, \text { for some } k_{0} \in \mathbb{N} \text { and } \inf _{n} \frac{n}{b_{n}}>0
$$

we have

$$
\mathcal{I}_{<q}-\lim a_{n} b_{n}=0 .
$$

Proof. Again, we proceed by contradiction. Then there exists $\varepsilon>0$ such that $A_{\varepsilon}=\left\{n \in \mathbb{N}: a_{n} b_{n} \geq \varepsilon\right\} \notin \mathcal{I}_{<q}$, thus $\lambda\left(A_{\varepsilon}\right) \geq q$. For each $k \in \mathbb{N}$ ( as well for $k_{0}$ ) we have $q_{k}<q \leq \lambda\left(A_{\varepsilon}\right)$, and so

$$
\sum_{n \in A_{\varepsilon}} \frac{1}{n^{q_{k}}}=\infty
$$

Further the proof continues by the same way as it was outlined in Theorem 4.1.

Theorem 4.5. Let $0<q \leq 1$ and $\left(q_{k}\right)$ be a strictly increasing sequence of positive numbers which is convergent to $q$. If for some admissible ideal $\mathcal{I}$ holds

$$
\mathcal{I}-\lim a_{n} b_{n}=0
$$

for every sequences $\left(a_{n}\right),\left(b_{n}\right)$ of positive numbers such that

$$
\sum_{n=1}^{\infty} a_{n}^{q_{k_{0}}}<\infty \text { for some } k_{0} \in \mathbb{N} \text { and } \sup _{n} \frac{n}{b_{n}}<\infty
$$

then

$$
\mathcal{I}_{<q} \subseteq \mathcal{I}
$$

Proof. Let us assume that for any admissible ideal $\mathcal{I}$ we have $\mathcal{I}-\lim a_{n} b_{n}=0$ and take an arbitrary $M \in \mathcal{I}_{<q}$. It is sufficient to prove that $M \in \mathcal{I}$. Since $M \in \mathcal{I}_{<q}$ we have $\lambda(M)<q$ and so there exists a sufficiently large $k_{0} \in \mathbb{N}$ such that $\lambda(M)<q_{k_{0}}<q$. So

$$
\sum_{n \in M} \frac{1}{n^{q_{k_{0}}}}<\infty
$$

Again, the proof continues by the same way as it was outlined in Theorem 4.2. 
The above results (Theorem 4.4 and Theorem 4.5) allow us to give a characterization for the class $T_{q_{k}}^{q}\left(a_{n}, b_{n}\right)$.

Theorem 4.6. Let $0<q \leq 1$ and $\left(q_{k}\right)$ be a strictly increasing sequence of positive numbers which converges to $q$. Let $\left(a_{n}\right),\left(b_{n}\right)$ be positive real sequences. Then the class $T_{q_{k}}^{q}\left(a_{n}, b_{n}\right)$ consists of all admissible ideals $\mathcal{I} \subset 2^{\mathbb{N}}$ such that $\mathcal{I} \supseteq \mathcal{I}_{<q}$.

\section{Summary and scheme of main results}

Let $\left(a_{n}\right),\left(b_{n}\right)$ be fix sequences of positive real numbers having the appropriate property (3.1), (4.1) and (4.3), respectively. Denote in short classes given above $T\left(\alpha, \beta, a_{n}, b_{n}\right)=T(\alpha, \beta), T_{q}^{q_{k}}\left(a_{n}, b_{n}\right)=T_{q}^{q_{k}}$ and $T_{q_{k}}^{q}\left(a_{n}, b_{n}\right)=T_{q_{k}}^{q}$. Then we have

i) for $0<\alpha \leq 1 \leq \beta \leq \frac{1}{\alpha}$,

$$
T(\alpha, \beta)=\left\{\mathcal{I} \subset 2^{\mathbb{N}}: \mathcal{I} \text { is admissible ideal such that } \mathcal{I} \supseteq \mathcal{I}_{c}^{(\alpha \beta)}\right\},
$$

ii) for $1 \geq q_{k}>q \geq 0(k=1,2 \ldots), q_{k} \downarrow q$ as $k \rightarrow \infty$,

$$
T_{q}^{q_{k}}=\left\{\mathcal{I} \subset 2^{\mathbb{N}}: \mathcal{I} \text { is admissible ideal such that } \mathcal{I} \supseteq \mathcal{I}_{\leq q}\right\},
$$

iii) for $0<q_{k}<q \leq 1(k=1,2 \ldots), q_{k} \uparrow q$ as $k \rightarrow \infty$,

$$
T_{q_{k}}^{q}=\left\{\mathcal{I} \subset 2^{\mathbb{N}}: \mathcal{I} \text { is admissible ideal such that } \mathcal{I} \supseteq \mathcal{I}_{<q}\right\} .
$$

For special cases the following scheme shows the smallest(minimal) admissible ideals partially ordered by inclusion which belong to the classes in the second line.

$$
\begin{aligned}
& \mathcal{I}_{0} \subsetneq \mathcal{I}_{c}^{(\alpha \beta)} \subsetneq \mathcal{I}_{<q} \quad \subsetneq \quad \mathcal{I}_{c}^{(q)} \quad \subsetneq \quad \mathcal{I}_{\leq q} \quad \subsetneq \quad \mathcal{I}_{<1} \quad \subsetneq \quad \mathcal{I}_{c}^{(1)} \\
& \uparrow \uparrow \uparrow \uparrow \uparrow \uparrow \uparrow \\
& T_{0}^{q_{k}} \supsetneq \underset{\text { if } \alpha \beta<q}{T(\alpha, \beta)} \supsetneq T_{q_{k}}^{q} \supsetneq \underset{\text { if } \alpha \beta=q}{T(\alpha, \beta)} \supsetneq T_{q}^{q_{k}} \supsetneq T_{q_{k}}^{1} \supsetneq \underset{\text { if } \alpha \beta=1}{T(\alpha, \beta)}
\end{aligned}
$$

\section{References}

[1] V. Baláž, J. Gogola, T. Visnyai: $\mathcal{I}_{c}^{(q)}$-convergence of arithmetical functions, J. Number Theory 183 (2018), pp. 74-83, DOI: http://dx.doi.org/10.1016/j.jnt.2017.07.006.

[2] V. Baláž, O. Strauch, T. Šalát: Remarks on several types of convergence of bounded sequences, Acta Math. Univ. Ostraviensis 14 (2006), pp. 3-12.

[3] N. Burbaki: Éléments de Mathématique, Topologie Générale Livre III, (Russian translation) Obščaja topologija. Osnovnye struktury, Moskow: Nauka, 1968. 
[4] A. Fasaint, G. Grekos, L. Mišík: Some generalizations of Olivier's Theorem, Math. Bohemica 141 (2016), pp. 483-494, DOI: https://doi.org/10.21136/MB.2016.0057-15.

[5] H. FAst: Sur la convergence statistique, Colloq. Math. 2 (1951), pp. 241-244.

[6] H. Furstenberg: Recurrence in Ergodic Theory and Combinatorial Number Theory, Princeton: Princeton University Press, 1981.

[7] J. Gogola, M. Mačaj, T. Visnyai: $O n \mathcal{I}_{c}^{(q)}$ - convergence, Ann. Math. Inform. 38 (2011), pp. $27-36$.

[8] K. Knopp: Theorie und Anwendung unendlichen Reisen, Berlin: Princeton University Press, 1931.

[9] P. Kostyrko, M. MaČAJ, T. ŠAlát, M. Sleziak: $\mathcal{I}$-convergence and extremal $\mathcal{I}$-limit poits, Math. Slovaca 55 (2005), pp. 443-464.

[10] P. Kostyrko, M. MaČaj, T. Šalát, O. Strrauch: On statistical limit points, Proc. Amer. Math. Soc. 129 (2001), pp. 2647-2654, DOI: https://doi.org/10.1090/S0002-9939-00-05891-3.

[11] P. Kostyrko, T. Š Alát, W. Wilczyński: $\mathcal{I}$ - convergence, Real Anal. Exchange 26 (2000), pp. 669-686.

[12] C. P. Niculescu, G. T. PrÃJitură: Some open problems concerning the convergence of positive series, Ann. Acad. Rom. Sci. Ser. Math. Appl. 6.1 (2014), pp. 85-99.

[13] L. Olivier: Remarques sur les séries infinies et leur convergence, J. Reine Angew. Math. 2 (1827), pp. 31-44, DOI: https://doi.org/10.1515/crll.1827.2.31.

[14] G. Pólya, G. Szegõ: Problems and Theorems in Analysis I. Berlin Heidelberg New York: Springer-Verlag, 1978.

[15] J. Renling: Applications of nonstandard analysis in additive number theory, Bull. of Symbolic Logic 6 (2000), pp. 331-341.

[16] T. ŠAlát, V. Toma: A classical Olivier's theorem and statistical convergence, Ann. Math. Blaise Pascal 1 (2001), pp. 305-313.

[17] I. J. Schoenberg: The Integrability of Certain Functions and Related Summability Methods, Amer. Math. Monthly 66 (1959), pp. 361-375.

[18] H. Steinhaus: Sur la convergence ordinaire et la convergence asymptotique, Colloq. Math. 2 (1951), pp. 73-74.

[19] J. T. Tóth, F. Filip, J. Bukor, L. Zsilinszky: $\mathcal{I}_{<q}-$ and $\mathcal{I}_{\leq q}-$ convergence of arithmetic functions, Period. Math. Hung. (2020), to appear, DOI: https://doi.org/10.1007/s10998-020-00345-y. 